\title{
Alternate architecture for the Origins Space Telescope
}

\author{
Jonathan W. Arenberg $\odot$, John Pohner, Michael Petach, Ryan Hallø, \\ Jeffrey Bautista, Mark Michaelian, and Tanh Nguyen \\ Northrop Grumman Space Systems, Redondo Beach, California, United States
}

\begin{abstract}
We report on our investigation into adapting the design of the James Webb Space Telescope (JWST) to the needs and requirements of the Origins Space Telescope. The modifications needed to the equipment and insulation of the JWST design to achieve the 4.5-K design temperature for Origins are introduced and detailed. The Webb thermal model is modified to the Origins design and used to predict the heat loads at 18 and $4.5 \mathrm{~K}$. We also describe the needed development of the JWST Mid-Infrared Instrument's cryocooler needed to reach the temperature necessary for Origins. The capabilities of the various modified cryocoolers are discussed. We show that three modified coolers are needed to achieve the performance required for Origins. Finally, we show that the baseline instruments and needed coolers can be accommodated for volume, mass, and power in the Webb architecture. (C) The Authors. Published by SPIE under a Creative Commons Attribution 4.0 Unported License. Distribution or reproduction of this work in whole or in part requires full attribution of the original publication, including its DOI. [DOI: 10.1117/1.JATIS.7.1 .011006]
\end{abstract}

Keywords: Origins Space Telescope; James Webb Space Telescope; cryocoolers; far-IR missions; single aperture far-IR mission.

Paper 20073SS received Jun. 14, 2020; accepted for publication Jan. 12, 2021; published online Feb. 10, 2021.

\section{Introduction}

In order to perform its scientific mission, the Origins Space Telescope must have optics that operate at $4.5 \mathrm{~K}$ with instruments at or below this temperature. ${ }^{1}$ Given the challenges, real and perceived for large cryogenic missions such as Spitzer and the James Webb Space Telescope (JWST), realizing a mission like Origins might sound like engineering or science fiction. We do not subscribe to this world view and believe that large cryogenic missions are indeed possible. The primary objective of our report is to show that as exotic and hard as it sounds, a 4.5-K telescope in the size needed for Origins is realizable and technologically feasible. From the other articles in this special section, it is clear that the Origins mission does have a strong baseline design for the Origins observatory, telescope, and instruments. This report is intended to reinforce the conclusion of these studies, namely achieving a $4.5-\mathrm{K}, 6-\mathrm{m}$ class observatory is possible by showing there is another viable architecture. It should be noted that our study concentrates mainly on the thermal performance of the telescope optics and is not a complete mission study.

We provide our reinforcing argument to that of the mission study team by analyzing a modified design for the Webb telescope and showing that operational temperatures of $4.5 \mathrm{~K}$ can be reached for a finite and small number of coolers, similar to what the Origins study has found. ${ }^{1,2}$ We also define a path to modify current state-of-the art coolers to provide the necessary lift to reach $4.5 \mathrm{~K}$.

The foundation of our Origins telescope conceptual design uses the design of the JWST as a point of departure (see Fig. 1). We detail the modifications needed to transform the Webb telescope's design into a concept for Origins telescope.

We describe in some detail how to modify an existing mature cryocooler to the cooling needs of Origins requirements, underscoring the argument that reaching $4.5 \mathrm{~K}$ is a challenge of engineering design and not one of technology. This report concludes with a discussion of packing the hardware for our JWST-derived Origins design into the mass and volume allocation of JWST.

*Address all correspondence to Jonathan W. Arenberg, jon.arenberg@ngc.com 
A secondary objective of this work is to demonstrate that a well-considered telescope architecture, such as JWST, can be used with small changes to meet different science missions. The motivation here is quite simple: reuse the JWST to the extent possible, resulting in a $6.5-\mathrm{m}$ diameter primary to satisfy the needs of Origins. A mission requiring a cryogenic payload such as Origins can reuse the investments made in realizing the JWST sunshield. If the basic architecture and some of the hardware are reused, the JWST-derived Origins mission would avoid much of the non-recurring expenses involved in the design of the original mission. Large-scale design reuse is one means of achieving the increase in engineering productivity necessary to maintain the viability of strategic missions under a fixed budget. ${ }^{3,4}$

In some respects, this study is deja vu. A previous decadal study called Single Aperture Far Infra-Red (SAFIR), another large-aperture cryogenic mission, pursued the same path of concept development as we are investigating. This path is adapting the JWST design for the Far-IR mission with the addition of active cooling. ${ }^{5,6}$ The SAFIR study was performed in the early 2000s, long before JWST's Critical Design Review, which was held in 2010. The SAFIR study made two seminal assumptions: the first is maturation of the planned cryocoolers for JWST's MidInfra-Red Instrument (MIRI), and the second is the success of the JWST design. At the time of this writing, in 2020, both of these design assumptions have been validated; JWST is in final system preparations for launch and the MIRI cryocooler is integrated and on board. This paper is a more detailed and nuanced repetition of the analysis performed for SAFIR, based on validated flight JWST thermal models and the defined Origins instruments.

In adapting the Webb telescope design for Origins, we have taken a step-by-step approach. The first step in this process is to realign the Webb design to the challenge of the Origins mission, while changing as little of the existing design as possible. The modifications to the Webb architecture and design are discussed and detailed in Sec. 2. Our Webb-based Origins telescope design is then implemented into a system-level thermal model derived from the validated Webb system thermal model that is used to calculate temperatures and heat loads. The locations in the design where heat will be actively removed are identified and the heat loads at those points calculated. Summing over those loads informs the total heat loads to be removed and determines the number of coolers needed.

The Webb cryocooler, originally designed for the MIRI instrument, achieves $\sim 6.2 \mathrm{~K}$ at the cold head. ${ }^{7}$ We extensively discuss the clear maturation path to extending Northrop Grumman's MIRI cooler to $4.5 \mathrm{~K}$ operation in Sec. 3.

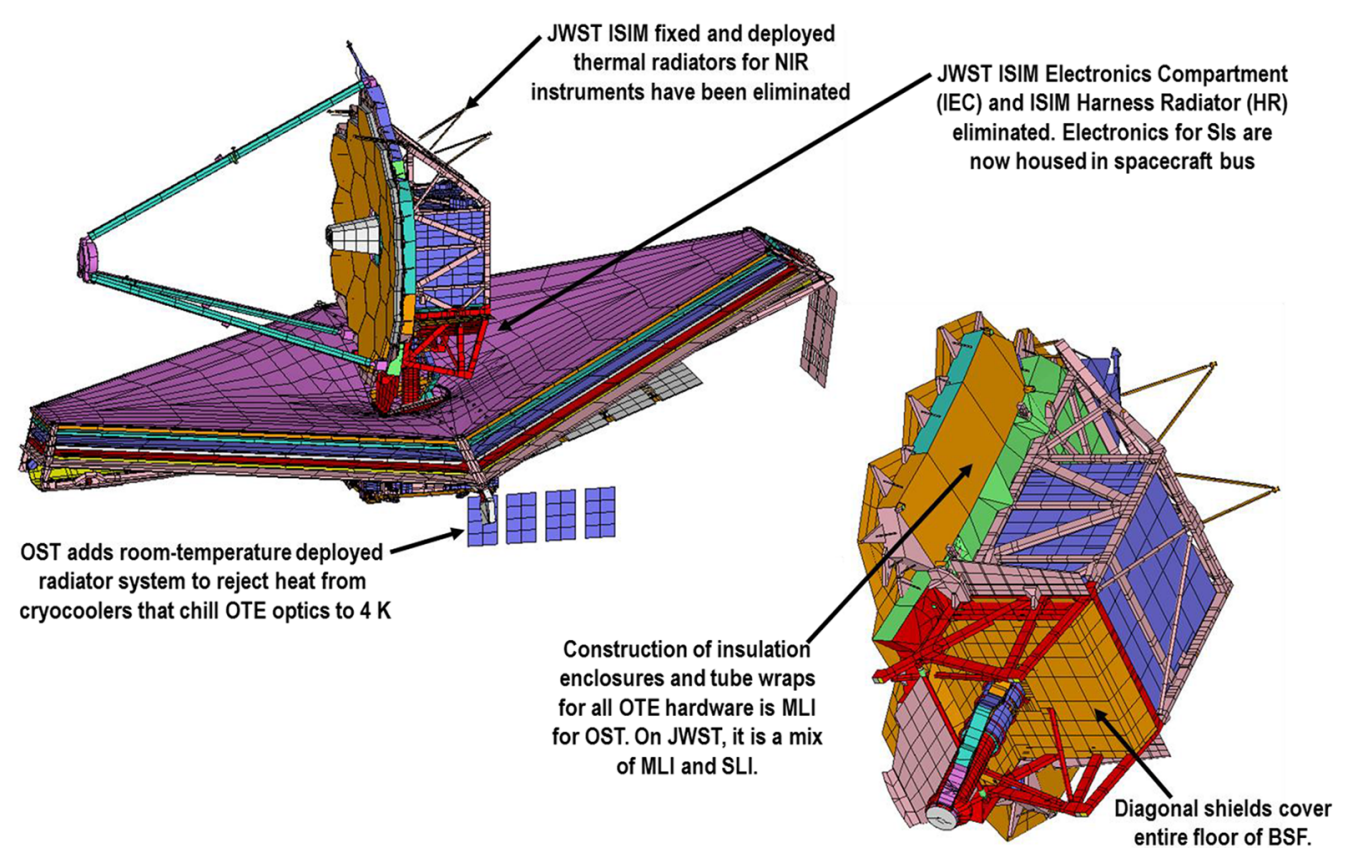

Fig. 1 View of the JWST derived Origins architecture. 
In Sec. 4, we show the packaging needed to accommodate the Origins equipment, coolers, cooler radiators, and instruments in the existing JWST volumes. Finally, we show that the mass properties of the redesigned Webb continue to meet launch and momentum management needs, implicitly making the argument that the resulting design can be launched and operated in the Sun Earth L2 halo orbit as planned for Webb and Origins.

\section{Changes to Transform Webb Telescope into the Origins Telescope}

This section describes the changes made to the Webb telescope configuration to allow it to meet the thermal requirements of the Origins telescope. This reconfiguration was also done with the subordinate objective of making the fewest changes to the Webb design. The "genetic proximity" of the two designs maximizes the reuse of design is at the foundation of demonstrating that a well-considered space observatory architecture can be (largely) re-employed to serve other science missions, thus providing a path to increased engineering productivity and reduced mission development times. ${ }^{4}$

\subsection{Architectural Changes Transforming Webb into Origins}

Figure 2 shows a picture of the proposed configuration and identifies the major changes to the Webb architecture to transform it into a design for Origins.

The primary change from JWST to Origins is the addition of active cooling, the result of which is telescope optics at $4.5 \mathrm{~K}$. The low operating temperature of the Origins telescope is the foundation of a vast increase in sensitivity that is the hallmark of the Origins concept. Thus, achieving these mirror temperatures is our design's chief objective. ${ }^{1}$ The $4.5-\mathrm{K}$ operating temperature cannot be achieved through a passive design. Achieving $4.5 \mathrm{~K}$ for the mirrors and instrument interfaces requires the addition and accommodation of a number of cryocoolers. A collateral goal of the Origins design effort is to limit the number of cryocoolers needed, thus minimizing the resources needed to make Origins feasible.

To provide an image of the thermal system, consider Fig. 2, a thermal schematic representation of a cooled system. It illustrates the design parameters of the cooling problem: discrete thermal stages or zones characterized by a specific temperature $T_{i}$, the amount of heat to be removed at each stage $Q_{r_{i}}$, and the heat conducted and radiated into the $i$ 'th node or stage of the system $Q_{\text {ext }_{i}}$. The specific power (SP) and specific mass (SM) are defined as the power or mass of a cooler needed to extract a watt of power at a given temperature $T$. For cryocoolers, SP and SM are typically represented as power laws and have the form

$$
\mathrm{SP}_{i}=\mathrm{AT}_{i}^{\alpha}
$$

for specific power. Figure 3 shows a plot of the known cryocoolers for space and shows the SP curve. SM is represented by

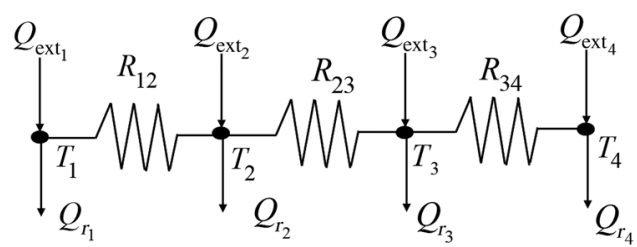

- $Q_{\text {ext }}$ External heat source, node $i$

- $T_{i}$ node $i$ temperature

- $Q_{r_{j}}$ Removed heat, node $i$

- $R_{i j}$ thermal resistance between nodes $i$ and $j$

Fig. 2 Schematic thermal representation of a multi-stage system. 


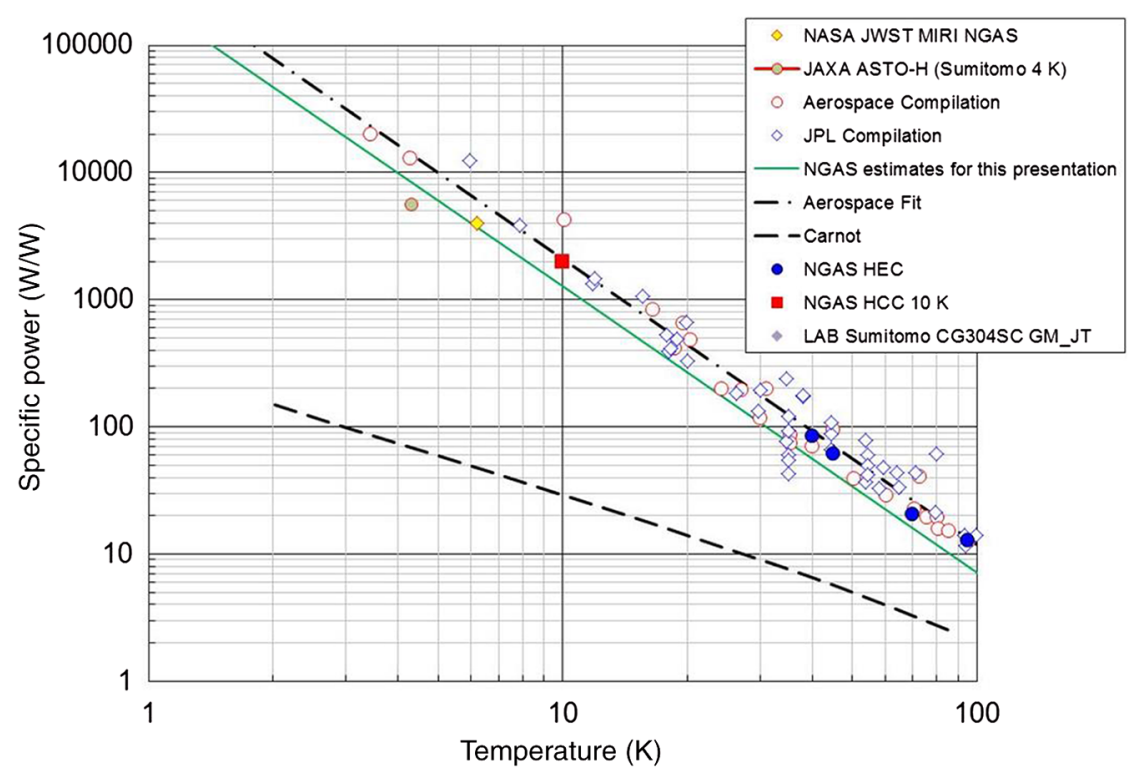

Fig. 3 Plot of specific power for space cryocoolers.

$$
\mathrm{SM}_{i}=\mathrm{BT}_{i}^{\beta}+\gamma
$$

In Eq. (2), $\gamma$ represents the irreducible mass for any cooler regardless of temperature. The system optimization is to minimize system power and mass while achieving the required temperature at $T_{4}$. Writing out the cooling system mass $M$ and power $P$ yields

$$
M=\sum_{i} Q S M_{i} \delta_{i}
$$

for mass and for power

$$
P=\sum_{i} Q S P_{i} \delta_{i}
$$

In Eqs. (3) and (4), $Q$ number of coolers and $\delta_{i}$ has the value of 0 if there is no active cooling for the $i$ 'th stage and 1 is there is active cooling. Equations (1)-(4) express the thermal system design problem. The designer must determine the number of stages and the temperatures of these stages to minimize overall power $P$ and mass $M$ of the cooling system. Figure 3 shows a plot of specific power for space cryocoolers and shows that $\alpha \sim-2.25$ implying that simply removing heat only at the coldest point is not a good option for the design of an active cooling system like Origins. System optimization is not simply a matter of increasing $Q_{r_{4}}$ until $T_{4}$ is reached; it requires a careful strategy of removing heat at higher temperatures where it can be removed more efficiently. The Origins architecture seeks to thermally isolate the coldest stage, the mirrors, limiting the heat that must be removed by the cryocoolers at the lowest temperature, thereby minimizing their number and required power, mass, and volume needed for cooler accommodation.

The key design change from the JWST to Origins is moving the warm (near-room temperature) instrument electronics from the cold side of the observatory. On Webb, the warm instrument electronics are located on the cold side of the observatory, proximate to the optics. This architecture presented a major challenge for the thermal design of JWST. Moving these warm electronics to the spacecraft is a critical design modification of the Webb design needed for Origins. Our Origins design places the warm-side instrument electronics in the spacecraft bus, physically well removed and isolated from the coldest areas of the observatory, thus removing the major source of conducted and radiated parasitic heat on the cold side of the Webb design. We also removed from the parent Webb design the harness radiator, instrument harnesses, and instrument radiators fixed and deployed, as they are not required in the Origins design. 
To minimize parasitic heat loads to the coldest stage, the JWST insulation on the instrument enclosure and backplane, which is mixture of MLI and black Kapton SLI, is replaced on our Origins design. The insulation for the Origins design completely covers the instrument volume with high-performance MLI to minimize refrigerant loads for instruments. For Origins, the backplane is covered with MLI with low- $\varepsilon$ surfaces facing the mirrors, limiting radiative coupling between the structure and mirrors. The JWST insulation on the Aft Optics Subsystem (AOS) is also replaced. For Webb, the AOS is covered with black Kapton SLI with Kevlar mesh, in our Origins design, the AOS is insulated with MLI to better thermally isolate AOS. Origins cools the fine steering mirror (FSM) and the tertiary mirror using heat straps connected to a $4.5-\mathrm{K}$ cold finger, replacing the AOS radiators employed in the Webb design.

The JWST wiring harness, which runs the full extent of the telescope from the room temperature spacecraft to the mirrors, is attached to the backplane and makes use of copper conductors. In the Webb design, the use of low-thermal-conductance phosphor bronze $(\mathrm{PhBr})$ is largely restricted to the transition harness running from the bottom to the top of the deployable tower assembly (DTA), where harness temperature gradients are the largest on Webb. The Origins harness would employ only $\mathrm{PhBr}$ or other low-thermal-conductance material throughout the harnesses. The exclusive use of $\mathrm{PhBr}$ in the harness will drastically lower the conducted parasitic heat load throughout our Origins design.

On JWST, the DTA surfaces are bare, in the Origins design the surfaces are covered with MLI and low- $\varepsilon$ surfaces to prevent heat from the $300-\mathrm{K}$ vibration isolator assembly, near base of DTA, from radiating into the cold side.

\subsection{Thermal Stages and Loads}

The validated JWST thermal model has over 118,000 nodes and was modified as described in Sec. 2.1 to represent our Origins design. With this model, we calculated the temperatures and heat flows through the Origins design. Our Origins design has two temperature stages or temperature intercepts at 18 and $4.5 \mathrm{~K}$. At each intercept, the heat is removed by active refrigeration by the cryocoolers the loads at each of these locations is determined by the thermal model.

The 18-K heat loads are considered first. The surfaces shown in yellow on the backplane support fixture in Fig. 4 are cooled to $18 \mathrm{~K}$ to reduce the required cooling load for the telescope

\begin{tabular}{|c|c|c|}
\hline Zone & Benefits & Unmargined 18K Heat Load [mW] \\
\hline A & ISIM & 1.5 \\
\hline B & ISIM & 8.0 \\
\hline C & ISIM & 83 \\
\hline D & ISIM & 1.1 \\
\hline E & ISIM & 6.3 \\
\hline F & ISIM & 4.8 \\
\hline G & PM/TM/FSM & 71.9 \\
\hline H & PM/TM/FSM & 30.1 \\
\hline I & ISIM & 36.2 \\
\hline J & PM/TM/FSM & 70.9 \\
\hline K & PM/TM/FSM & 27.3 \\
\hline L & ISIM & 27.5 \\
\hline & & Total $=203.9$ \\
\hline
\end{tabular}

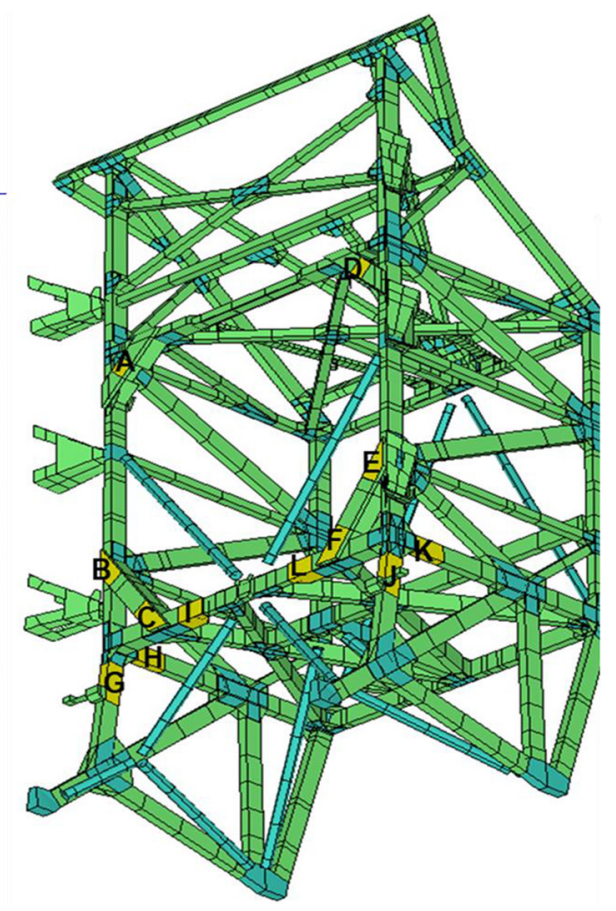

Fig. 4 18-K stage cooling locations. Specific locations and loads are indicated by the lettered points. 


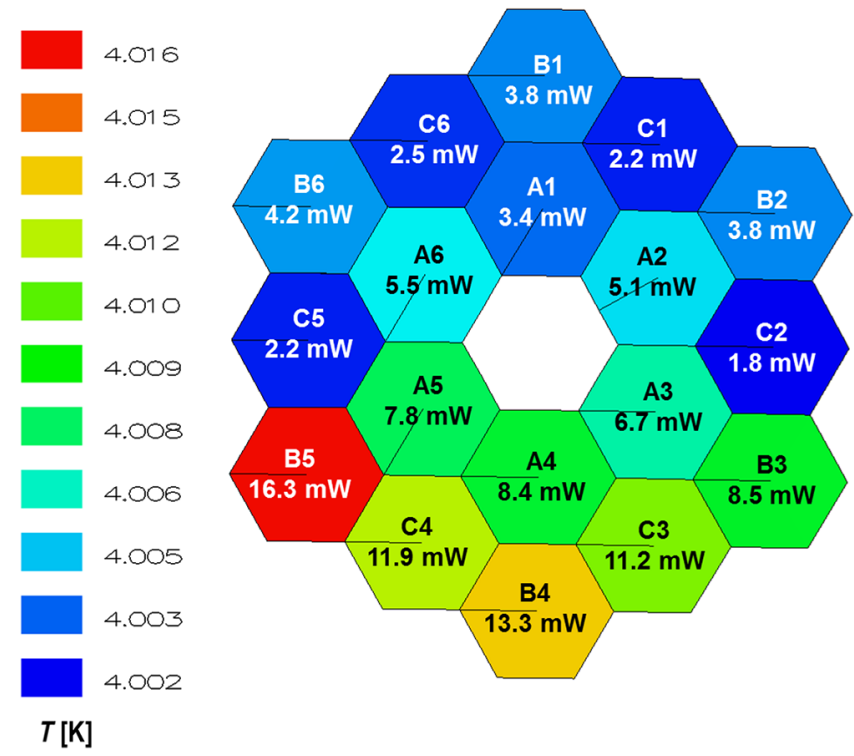

Fig. 5 Primary mirror heat loads.

optics and science instrument interfaces. This is done by transferring heat from these locations to a limited number of cold fingers using high-purity aluminum straps. The total heat to be removed from all locations on the telescope structure at $18 \mathrm{~K}$ is $203.9 \mathrm{~mW}$ with no additional margin (as yet) included.

Several other locations are also in the $18-\mathrm{K}$ stage. On Webb, all thermally significant harnesses that run from the warm spacecraft bus to the cold telescope must pass through an electrical interconnect panel called ICP6. These harnesses passing through ICP6 include those for controlling primary mirror segment assemblies (PMSA), SM, and FSM, as well as telescope deployments mechanisms and heaters, temperature sensors, etc. Cooling ICP6 to $18 \mathrm{~K}$ requires $339 \mathrm{~mW}$ power to be removed, larger than the telescope structure loads. Origins also calls for the cooling of the Cold Junction Box (CJB) located near ICP6, on underside of floor of the backplane support. The harnesses for controlling PMSAs, SM, and FSM pass through the CJB. Cooling CJB to $18 \mathrm{~K}$ helps augment cooling at $18 \mathrm{~K}$ locations and further reduces the heat load on mirrors operating at $4.5 \mathrm{~K}$ by removing potential parasitics at a higher temperature, where they are more efficiently removed. The predicted $18 \mathrm{~K}$ heat load on the CJB is $38 \mathrm{~mW}$.

Summing up all of the heat loads at $18 \mathrm{~K}$, including those from the structure $(204 \mathrm{~mW})$, ICP6 (339 mW), and the CJB $(38 \mathrm{~mW})$, produces a total of $581 \mathrm{~mW}$.

Let us now examine the $4.5-\mathrm{K}$ loads. The thermal loads on the optics are also calculated directly from the Origins thermal model. Figure 5 shows the heat loads on the primary mirror.

The total $4.5-\mathrm{K}$ load from the primary mirror, without margin, is $118.8 \mathrm{~mW}$, the loads on the individual mirror is shown in Fig. 5. The very noticeable "hot spot" in the mirror is due to an asymmetry in the design of the Webb harness. This hot spot is clearly an area that can be investigated for further improvement especially given the highly non-linear dependence of radiated stray light. We have investigated the performance of the PM as shown. Lightsey et al. ${ }^{8}$ provided an assessment of the stray light performance of this configuration.

The 4.5-K loads on the other telescope optics are $9.9 \mathrm{~mW}$ for the SM, $1.7 \mathrm{~mW}$ for the TM, and $4.7 \mathrm{~mW}$ for the FSM. This gives an unmargined total heat load of $135.1 \mathrm{~mW}$ at $4.5 \mathrm{~K}$ for the telescope optics.

\section{Cryocoolers}

The changes in configuration as described in Sec. 2 are necessary for the passively cooled $\sim 40 \mathrm{~K}$ Webb telescope to be capable of being actively cooled to the temperatures needed for Origins. To reach the $4.5-\mathrm{K}$ operating temperature requires active cooling, via cryocoolers. This section 
details the developments need and possible to extend the current start of the art to the levels of performance needed for Origins. The maturation of coolers is also the subject of companion paper in this special issue. ${ }^{9}$ Following the description of cryocooler development paths, we will present an analysis that determines the number of cryocoolers needed to meet the required loads defined in the previous section.

\subsection{Path to a 4.5-K Cooler}

The cryocoolers needed for Origins do not yet exist, their development is part of the planned activities. ${ }^{9,10}$ In this section, we detail our thoughts on the development of the MIRI cooler developed for JWST to meet the needs of Origins.

Our design paradigm of the maximum reuse of Webb architecture for Origins suggests that that the cryocooler that will be used for the MIRI instrument will be re-employed for Origins, meaning no entirely new cooler development is needed for Origins as was the case for Webb. The Webb MIRI cooler is designed to reach $6.25 \mathrm{~K}$ not $4.5 \mathrm{~K}$ and cannot be used to meet the needs of Origins design unmodified. The required lift for the Webb MIRI cooler is $55 \mathrm{~mW}$ at $6.25 \mathrm{~K}$ and $232 \mathrm{~mW}$ at $18 \mathrm{~K}$. The MIRI cryocooler is the state-of-the art and is our point of departure for a cooler for Origins.

Northrop Grumman has developed and manufactured a large number of space flight cryocoolers covering the temperature range from 6 to $200 \mathrm{~K} .{ }^{11}$ The cryocooler that we modeled in this study is based on modifications to the MIRI flight cooler manufactured by Northrop Grumman Space Systems for NASA's JWST shown in Fig. $6{ }^{12-14}$ As a result, the modeled cooler is a relatively mature Technology Readiness Level 6 (TRL 6) and includes major subsystems that are TRL 8 . The MIRI cryocooler was designed to cool a sensor and its shields to 6.2 and $18 \mathrm{~K}$, respectively. We will show that with minor modifications this cooler will meet the needs of Origins.

Figure 7 shows a block diagram of the MIRI cooler. The cooler is a hybrid pulse tube/Joule Thomson (JT) cooler. A three-stage pulse tube cooler precools the circulating helium gas for the lowest temperature JT stage. The three-stage pulse tube cooler was originally designed as a 10-K cooler that can also provide additional cooling at each of its three stages for other components including thermal shields. ${ }^{15}$ The pulse tube and JT coolers have independent working gases and independent gas volumes. For this study, no changes were made to the pulse tube precooler-all changes were made to the JT cooler.

The existing MIRI JT cooler uses the TRL 9 Northrop Grumman High Efficiency Cool (HEC) compressor with the addition of rectifying reed valves to pressurize and circulate the ${ }^{4} \mathrm{He}$ JT gas. It acts as a vibrationally balanced single compression stage compressor. The JT recuperators (R2, R3, and R4) through which the JT stage's pressurized helium flows are precooled at each of the three pulse tube stages. The precooled helium then passes through

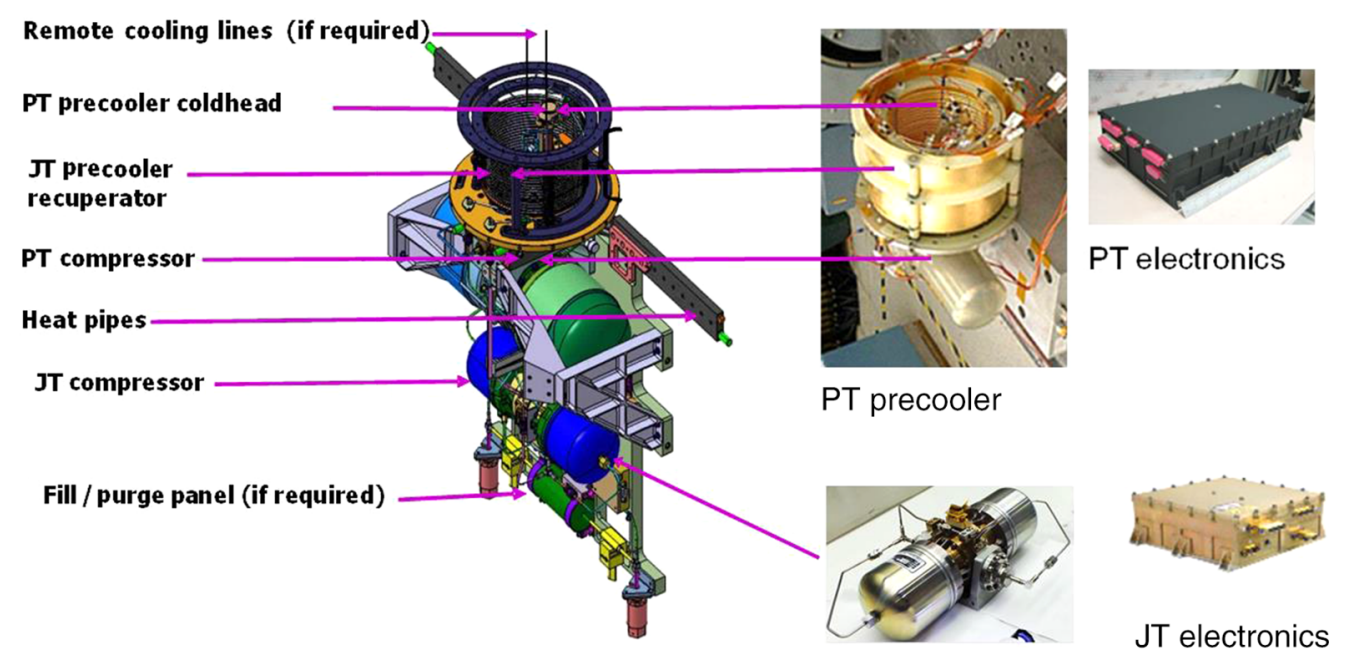

Fig. 6 The JWST MIRI cooler and its main components. 


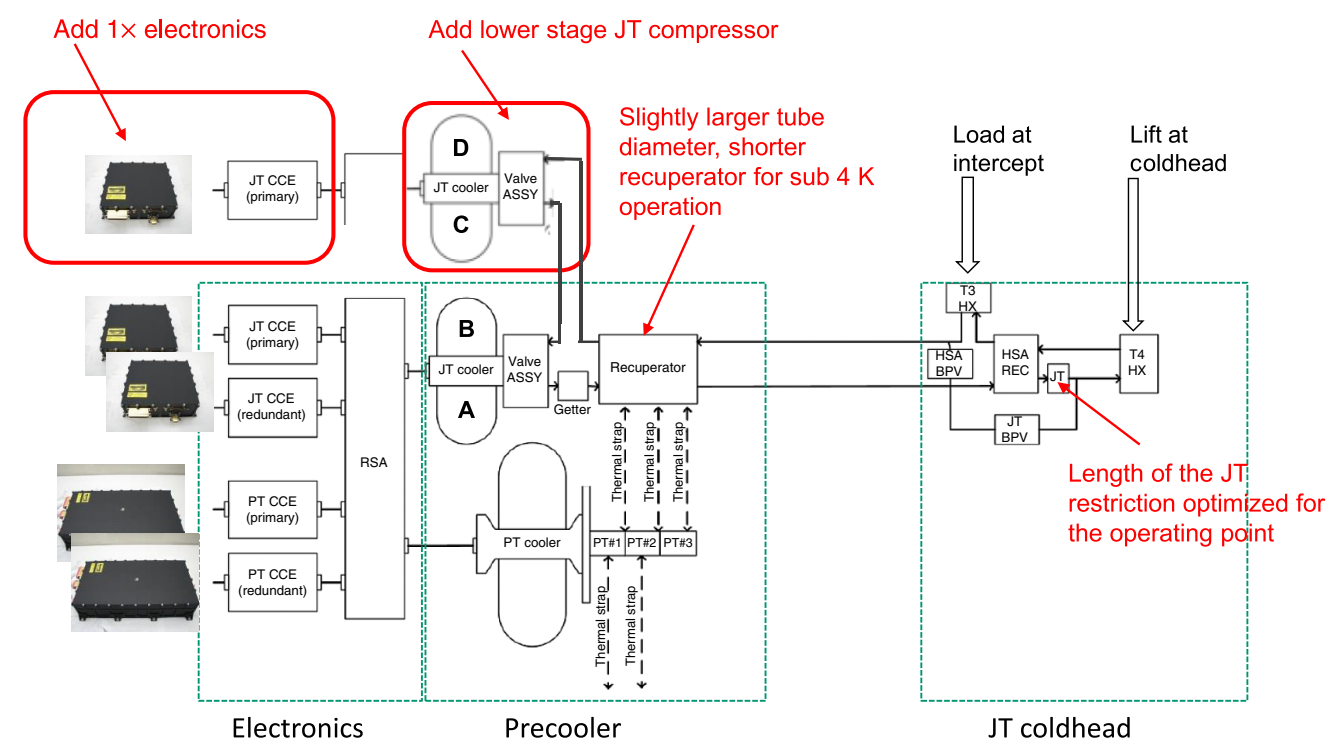

Fig. 7 Cryocooler block diagram, showing the modifications to allow significant lift at lower temperature.

the JT recuperator (R1) and is further cooled to $6 \mathrm{~K}$ at the JT expander. The bypass valve can be used to precool a large heat capacity load, if necessary. The fact that the JT cooling stage can be located many meters from the basic cooler as it is on MIRI is especially relevant to the cooling of a large reflector.

The MIRI cooler can be modified to operate efficiently at lower temperatures, as previously described, by adding another JT compressor to act as an additional compression stage, increasing the recuperator tube size modestly, and optimizing the length of the JT restriction. ${ }^{16}$ These modifications are indicated in the red text in Fig. 7. In the previous report, a point design for a large telescope cooled to $4.5 \mathrm{~K}$ was presented. ${ }^{16}$ In this current study, we extend this modeling to provide predictions of the performance of the modified MIRI cooler over a broad range of conditions suitable for supporting a range of potential future missions such as Origins.

For this modeling effort, we changed the operating conditions and/or changed the working fluid. In addition, we modeled changing the single-stage JT compressor to a multi-stage higher pressure ratio JT compressor. In all cases, we changed the operating conditions of the PT precooler in order to optimize precooling for the JT cooler but made no hardware changes to the PT precooler. For the JT cooler working fluid, we modeled the use of ${ }^{4} \mathrm{He}$ for temperatures above $4 \mathrm{~K}$ and the use of ${ }^{3} \mathrm{He}$ for temperatures below $4 \mathrm{~K}$ where it becomes advantageous.

The model used in this study was previously used in the development of the MIRI cooler and has been compared against measurements from that cooler. ${ }^{14}$ Both a detailed SAGE model and a reduced model were developed and compared against the measured performance of the MIRI cooler. For this work, the reduced model and NIST REFPROP 9 fluid properties were used. ${ }^{17}$

The current MIRI stage cooler was designed for cooling a primary load at $6.2 \mathrm{~K}$ with an additional upper stage heat exchanger for remotely intercepting parasitic loads at $18 \mathrm{~K}$. Figure 8 shows the predicted cooling versus input power of this configuration with its single-compression stage JT compressor when ${ }^{4} \mathrm{He}$ gas is used as the working gas for cooling at 6 and $18 \mathrm{~K}$. The model includes the power dissipation of the MIRI flight electronics, hence we report the power going into the cooler's drive electronics, i.e., the spacecraft bus power. Based on the 5\% to $10 \%$ difference between the model and measurements over the range of measurements shown in Fig. 8, we expect that the modeling results for the proposed configurations have a similar $\sim \pm 10 \%$ uncertainty band for the cases that use ${ }^{4} \mathrm{He}$ with higher uncertainty for the configurations that use ${ }^{3} \mathrm{He}$.

The curves with circle symbols are model predictions, the square symbols are measurements made with the MIRI cooler at an intercept load of $335 \mathrm{~mW}$ and correspond to the dashed line model predictions. 


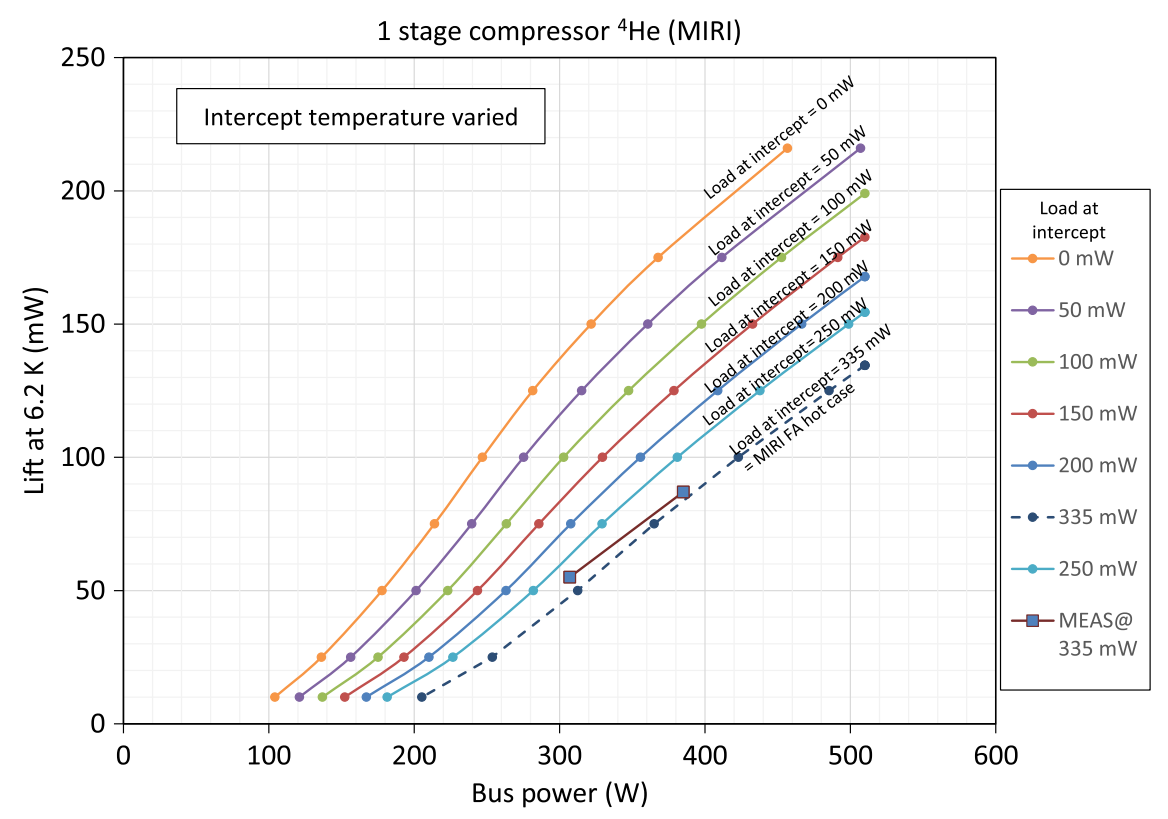

Fig. 8 Predicted lift at $6.2 \mathrm{~K}$ versus Bus Power when configured as MIRI cooler.

Next, we show the predicted performance of the cooler when we use ${ }^{4} \mathrm{He}$ and augment the existing single-stage JT compressor with an additional compressor stage with larger swept volume. This allows achieving the same mass flowrates as the MIRI cooler but with lower input pressures and a larger compression ratio, thus allowing lower temperature operation. For this configuration, we can use a design variant of the MIRI compressor that has larger pistons. We have previously demonstrated cooling at $4.5 \mathrm{~K}$ with the development model of the MIRI cooler, utilizing a laboratory compressor for the additional lower compression stage. ${ }^{18}$ Subsequently, we have developed to Critical Design Review level a flight version of the compressor with pistons that are approximately twice the diameter of the MIRI JT compressor. This new design variant has been originally developed for use in a heat engine application, then prototyped and advanced to released drawing status on subsequent NASA programs. ${ }^{19}$ Because of the development of this compressor for a thermoacoustic power convertor (TAPC), we call this the TAPC compressor. It uses the same motor and flexure design as the existing MIRI compressor, the only significant difference is the larger piston diameter. The TAPC compressor would need reed valves added to it to make it a JT compressor, similar to the prior addition of reed valves to the HEC pulse tube cryocooler compressor to make it a JT compressor. The MIRI reed valve design would be scaled to the larger area needed for the lower pressure operation. This is not expected to be a difficult development. The use of this new design variant of the JT compressor for the additional lower stages is the basis for the model predictions that follows.

There are two configurations in which we can utilize the TAPC design as an additional compressor. The simplest of these is to add it as a lower stage in the usual back-to-back configuration, which then results in a two-stage compression of the helium. Referring to Fig. 7, the first stage of compression is from the TAPC compressor sides " $\mathrm{C}$ " and " $\mathrm{D}$ " and the second stage is from the MIRI compressor sides "A" and "B." The predicted lift at $4.5 \mathrm{~K}$ for this two stage configuration is given in Fig. 9. The predicted lift shown in Fig. 9 is about 20\% greater than the measurements and predictions in the prior demonstration, which is because the current predictions allow the use of a JT restriction length that is optimized to the design point, as opposed to previously having to use the JT restriction hardware that was optimized for $6.2 \mathrm{~K}$ operation. With this configuration, the cooler is predicted to lift $157 \mathrm{~mW}$ at $4.5 \mathrm{~K}$ with no intercept load, or $97 \mathrm{~mW}$ lift at $4.5 \mathrm{~K}$ with $250 \mathrm{~mW}$ of load intercepted at $15 \mathrm{~K}$.

For additional lift at $4.5 \mathrm{~K}$, we can make each half of the TAPC compressor act as an independent compression stage, similar to the configuration flown by ESA on the Planck mission. ${ }^{20,21}$ Referring to Fig. 10, the first stage of compression is then from the TAPC compressor sides "D," the second stage of compression comes from the TAPC compressor side "C," 


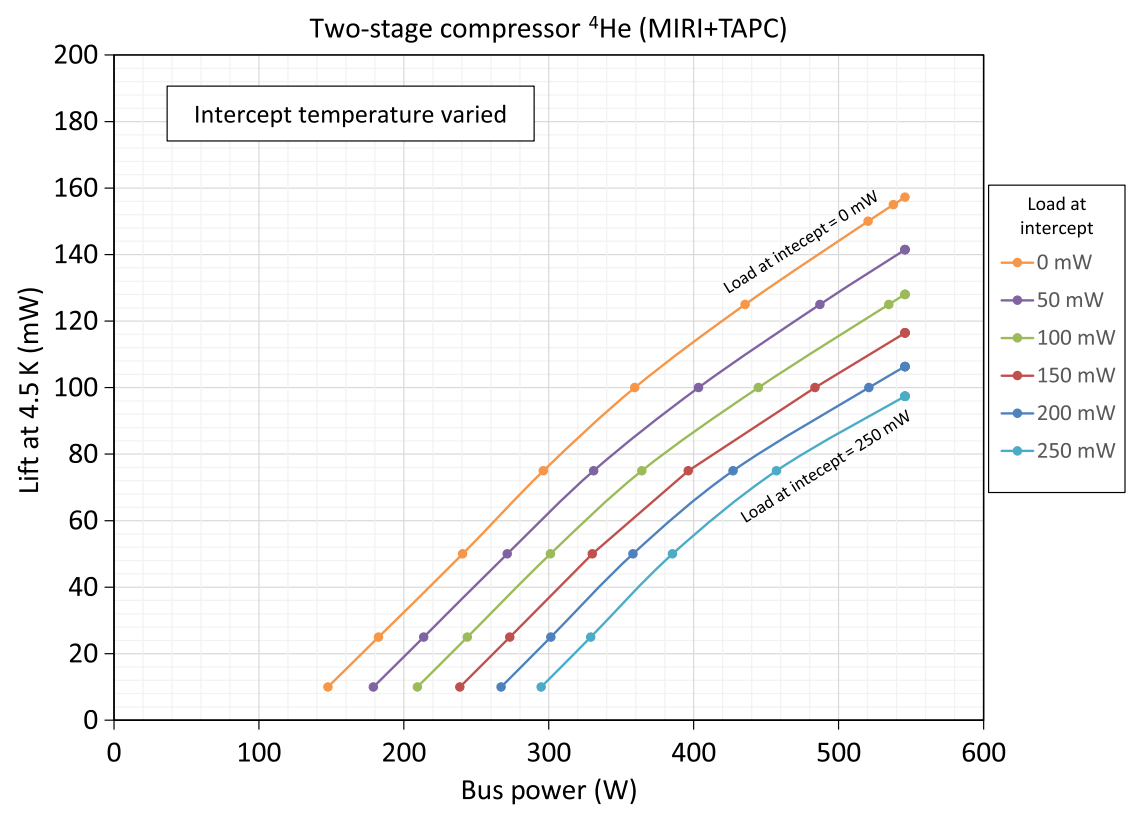

Fig. 9 Predicted heat lift at $4.5 \mathrm{~K}$ using MIRI cooler with two stage compression implemented via back-to-back TAPC compressor with ${ }^{4} \mathrm{He}$.

and the third stage of compression is from the back-to-back MIRI JT compressor sides "A" and "B" This provides three stages of compression but reduces the swept volume of the lowest stage by a factor of two. Figure 10 shows that this is an acceptable trade that results in a significant increase in lift at all bus powers. With this configuration, the cooler can lift $200 \mathrm{~mW}$ at $4.5 \mathrm{~K}$ with no intercept load, or $140 \mathrm{~mW}$ lift at $4.5 \mathrm{~K}$ with $250 \mathrm{~mW}$ of load intercepted at $18 \mathrm{~K}$.

Since the power split between the pulse tube precooler and the JT compressor can be varied by command, there are many possible combinations of intercept temperature, intercept load, and lift at $4.5 \mathrm{~K}$. To illustrate a representative set of conditions, we have modeled the case in which the intercept load is always four times the load at $4.5 \mathrm{~K}$, both for the case of a constant $18 \mathrm{~K}$

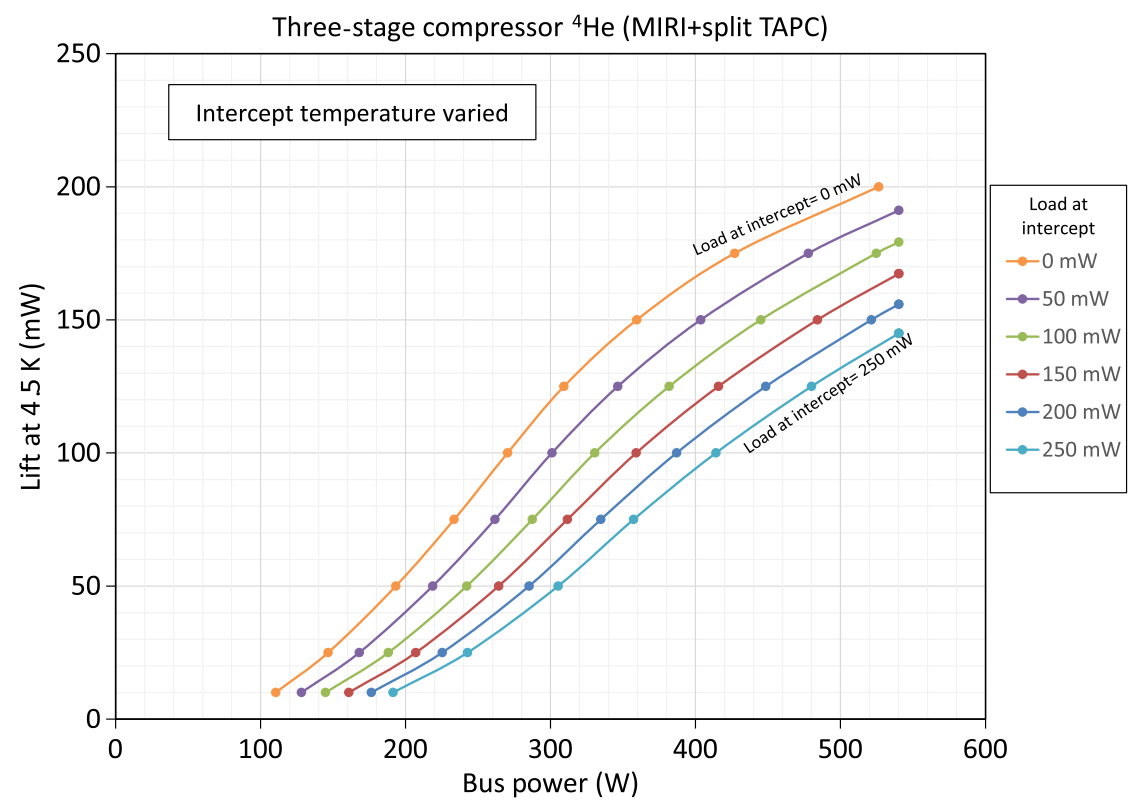

Fig. 10 Lift at $4.5 \mathrm{~K}$ for the configuration, in which the TAPC compressor is set up as two stages, and the MIRI compressor is used as the third stage, and the gas used is ${ }^{4} \mathrm{He}$. 


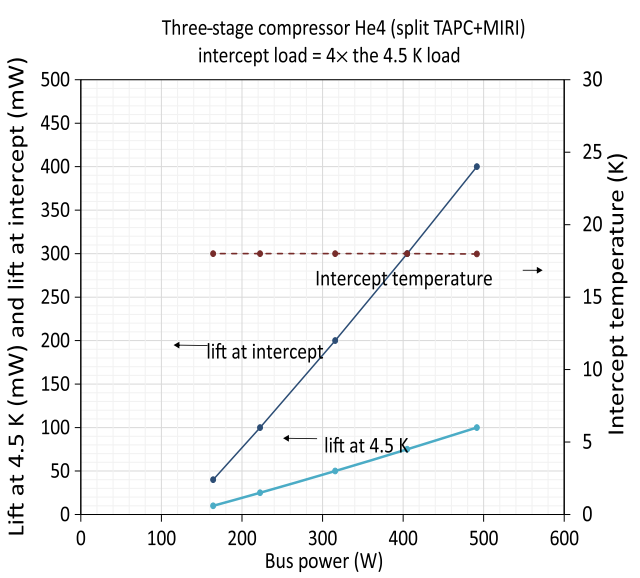

(a)

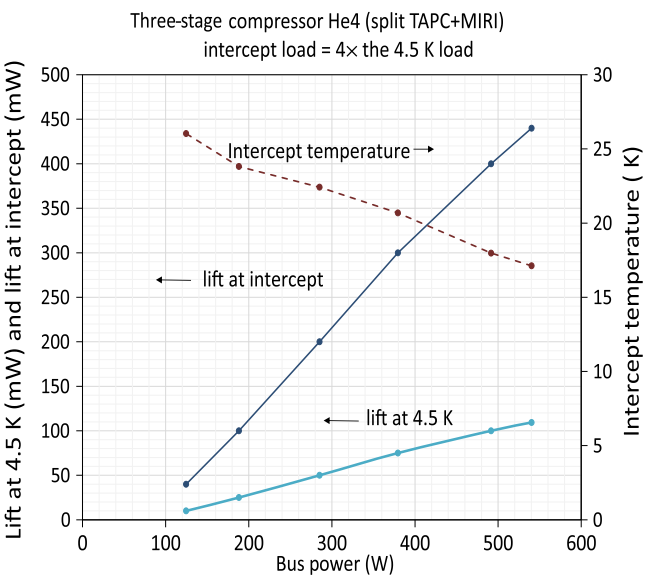

(b)

Fig. $114.5 \mathrm{~K}$ lift with three-stage compressor and ${ }^{4} \mathrm{He}$. Intercept load is 4 times the $4.5-\mathrm{K}$ load. (a) Constant intercept temperature of $18 \mathrm{~K}$ and (b) intercept temperature varied to minimize the bus power.

intercept temperature, and for the case where the intercept temperature is allowed to vary, so as to minimize the total power needed. These predicted results are shown in Fig. 11.

Lower temperatures can be reached by switching to ${ }^{3} \mathrm{He}$ from ${ }^{4} \mathrm{He}$ and reducing the operating pressure. Efficient operation at these low temperatures is achieved by splitting the two halves of the MIRI JT compressor into independent stages, which in conjunction with the split TAPC compressor provides four-stage compression. Referring to Fig. 7, the first stage of compression is from the TAPC compressor sides "D," the second stage from the TAPC compressor side "C," the third stage is from the MIRI compressor sides "B," and the fourth stage is the MIRI compressor sides "A". This four-stage compression not only accommodates the lower pressure, the resulting high compression ratios also mean that substantial cooling power is achieved at mass flowrates substantially lower than in the MIRI cooler application. This reduced mass flow rate allows recuperator lengths to be reduced by a factor of four, which helps to counteract the pressure drop in the recuperators that results from the lower gas density and lower operating pressures. To further accommodate the reduced pressure, the tubing diameter in the lower recuperator is increased by approximately two times relative to the MIRI cooler dimensions. The predicted lift in this configuration at 2.5 and $1.7 \mathrm{~K}$ is shown in Fig. 12.

The addition of the second compressor and its electronics is estimated to increase the mass of the cooler by $\sim 10 \mathrm{~kg}$.

A hybrid pulse tube/JT cryocooler based on the MIRI cooler can provide an effective solution for large cryogenic space missions that require significant cooling in the 4- to $2-\mathrm{K}$ range like Origins. The required modifications are confined to the addition of an existing design
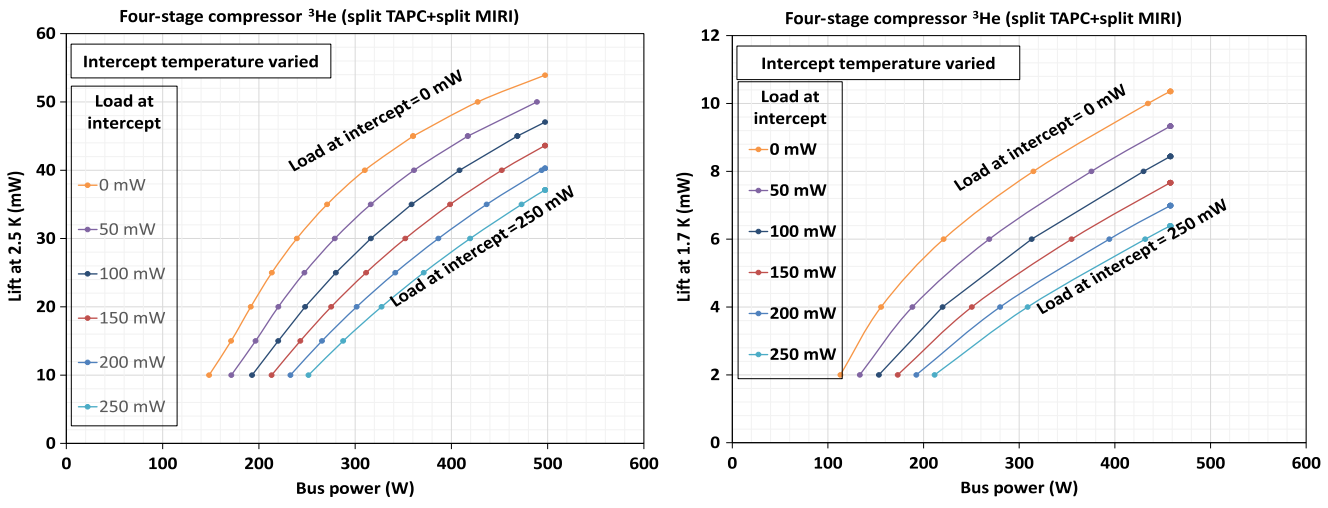

Fig. 12 Lift at 2.5 and $1.7 \mathrm{~K}$ with compressor configured for four compression stages, using ${ }^{3} \mathrm{He}$. 
Table 1 Cooler lift requirements versus capability for three-stage split TAPC + MIRI coolers.

\begin{tabular}{lccc}
\hline \hline Description & Requirement $(\mathrm{mW})$ & Capability $(\mathrm{mW})$ & Margin $(\%)$ \\
\hline Net load at $18 \mathrm{~K}$ & 581 & 1200 & 107 \\
Net load at $4.5 \mathrm{~K}$ & 173 & 300 & 73 \\
\hline \hline
\end{tabular}

compressor with its drive electronics and the resizing of the throttle. This approach reduces the hardware development needed for achieving the lift requirements of next generation space telescopes and can guide future demonstrations at correlated conditions in the lab.

\subsection{How Many Cryocoolers are Needed for Origins?}

In Sec. 2.1, we have developed an estimate of the load to cool the telescope to $4.5 \mathrm{~K}$. The required lift is $581 \mathrm{~mW}$ at $18 \mathrm{~K}$ and $136 \mathrm{~mW}$ at $4.5 \mathrm{~K}$. However, our analysis is not complete. The cooling needed to provide the $4.5-\mathrm{K}$ interface to the instruments has not been accounted for. At the time when we performed this quick conceptual study, the instrument loads were not well known. A companion paper in this issue from DiPirro and colleagues provides a basis for an estimate of the missing load at $4.5 \mathrm{~K} .{ }^{2}$ DiPirro reports that the total load for the baseline Origins design is $90 \mathrm{~mW}$. Examination of the heat flow diagram in the reference shows that much of this $90 \mathrm{~mW}, 53 \mathrm{~mW}$, are from the structure and telescope, leaving $37 \mathrm{~mW}$ as the estimated instrument load missing in our analysis.

Consulting Fig. 11, we can see that one three-stage split TAPC + MIRI configuration will lift $100 \mathrm{~mW}$ at $4.5 \mathrm{~K}$ and $400 \mathrm{~mW}$ at $18 \mathrm{~K}$ for $500 \mathrm{~W}$ of bus power. Our design calls for three of these coolers with a total lift at $4.5 \mathrm{~K}$ of $300 \mathrm{~mW}$ and $1200 \mathrm{~mW}$ at $18 \mathrm{~K}$ (Table 1).

The margins for this configuration are significant: over $100 \%$ for the $18-\mathrm{K}$ load and $73 \%$ for the 4.5-K load. We do believe that for a study of this type we have acceptable margins for a conceptual demonstration. Typically margins are decreased as the design and models mature, since our design paradigm is to reuse the Webb design, it is proper to consider it more mature than other conceptual models. Second, in our quick study, the target mirror temperature was $4 \mathrm{~K}$, not $4.5 \mathrm{~K}$, which induces some degree of conservatism. Since the heat load on the $4.5-\mathrm{K}$ optics is primarily conduction-dominated, the ratio of heat load on $4.5 \mathrm{~K}$ optics to that on the $4.0-\mathrm{K}$ optics 0.96 of that portion of the load. Finally, these margins are for a system with three coolers; the baseline Origins has four. The addition of a fourth cooler to this design would significantly overachieve insofar as thermal margin is concerned.

\section{Packaging}

The first objective of this packaging study was to demonstrate that the volume within the existing Webb design can accommodate the equipment for Origins in terms of volume, mass, and power.

The first task is to find volume in the spacecraft for the relocated instrument electronics. In the Webb configuration, the instrument electronics are located on the cold side of the sunshield. In the Webb design, heat from the single MIRI cooler is rejected on a radiator panel forming a side of the bus. This is the location planned for the instrument electronics.

The second objective of our packaging study is to find a means to reject the $1.5-\mathrm{kW}$ coming from the three cryocoolers needed for Origins. The needed power rejection is accomplished using a deployable room temperature radiator made of panels. Each panel measuring is $1.25 \mathrm{~m} \times$ $0.7 \mathrm{~m}$, with one panel per cooler. The ensemble of radiators is capable of rejecting nearly $1.6 \mathrm{~kW}$ at $290 \mathrm{~K}$. This radiator is located in the space now used for the MIRI in JWST and this is the initial location for the relocated instrument electronics. A sketch of this layout is given in Fig. 13. Figure 13 also indicates that there is room to grow the radiators in case increased margin is desired.

Volumetric accommodation of the science instruments was assessed by taking volume footprints of the existing Origins instruments and fitting them to the available volume in the JWST 


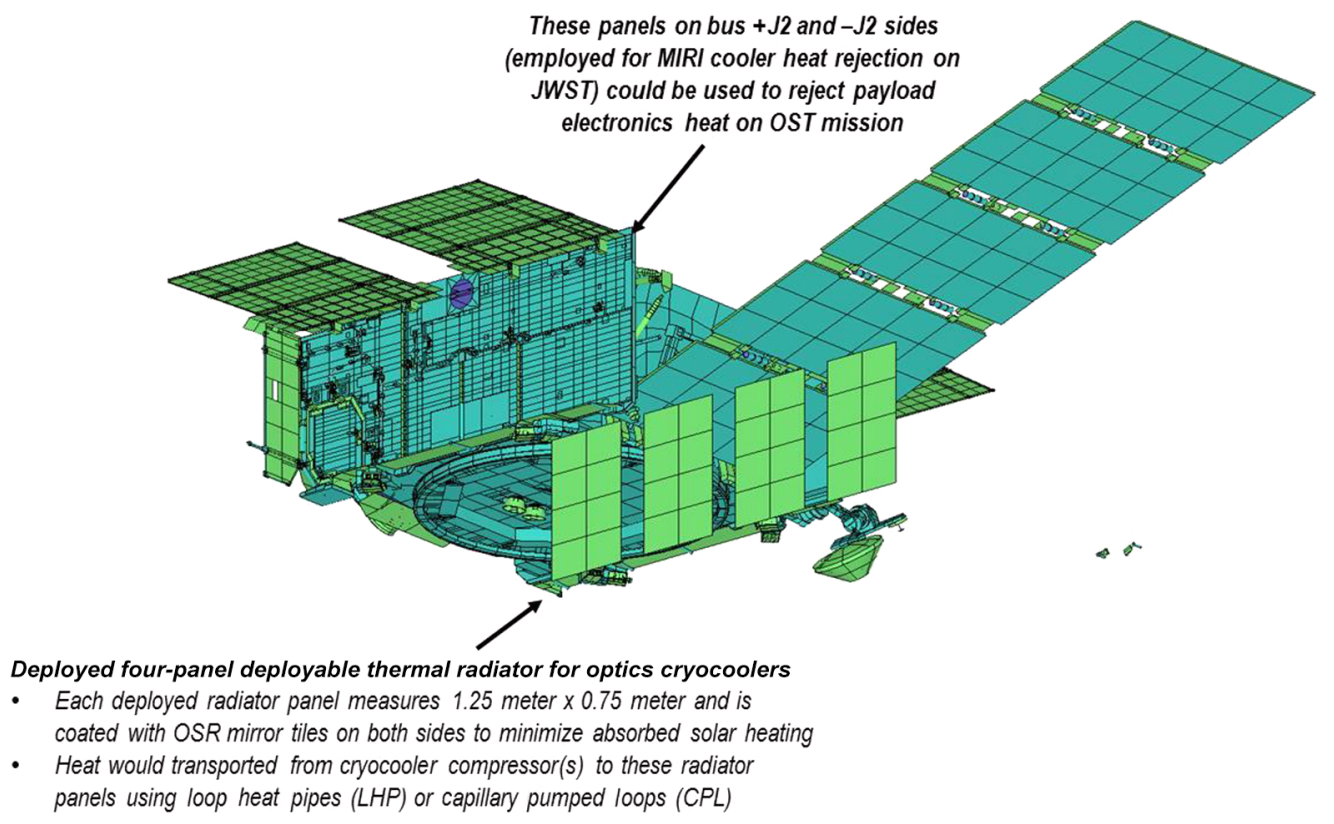

Fig. 13 Packaging for instrument and cryocooler electronics.

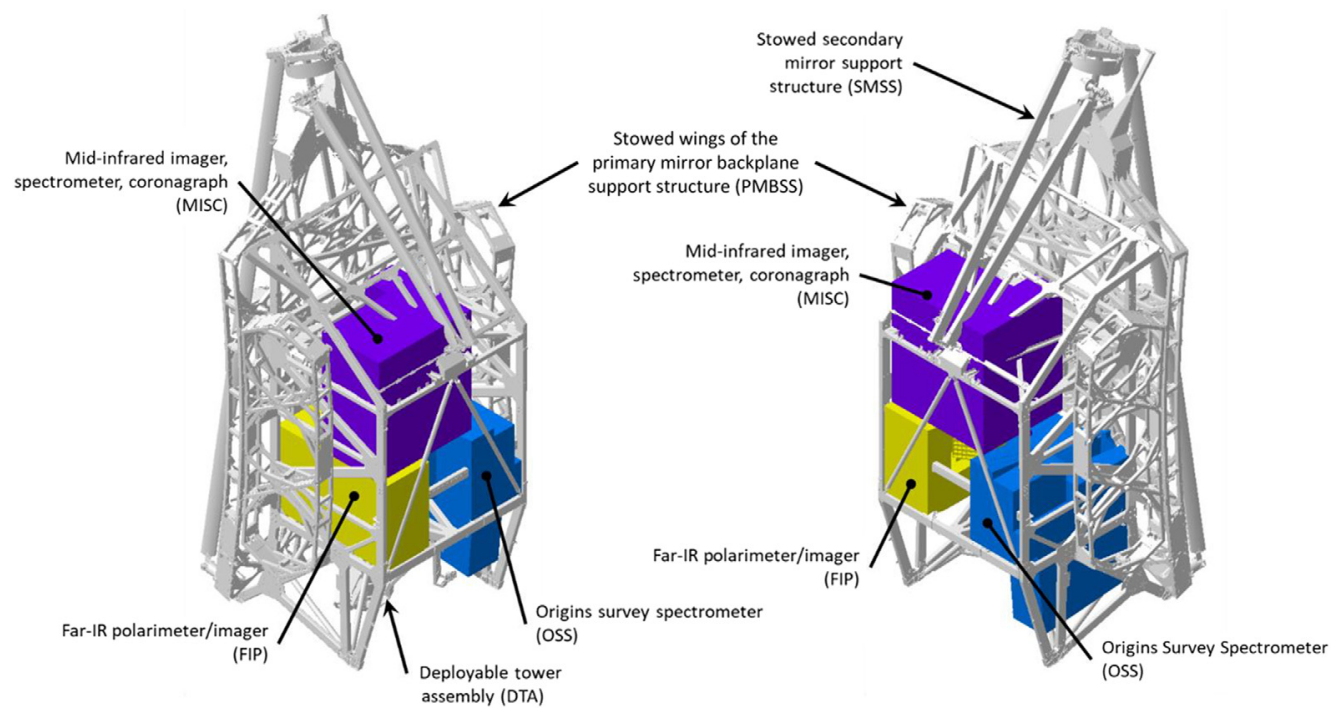

Fig. 14 Origins instruments FIP, OSS, and MISC, packaged in the ISIM and IEC volumes within the JWST structure.

ISIM and instrument electronics area. The results of the instrumentation packaging study are shown in Fig. 14. Basically, all of the instrument envelopes can be accommodated. There are few areas of interference with current backplane struts and the Origins instrument envelopes. These small interferences are not viewed as "show stoppers," as the instruments are represented by their envelopes and these envelopes were developed for the Origins baseline, not this Webb-derived architecture. Given the small size of the interferences, we are quite confident a deliberate effort to accommodate the Origins instruments would be successful and not upset the architecture.

We also examined the electrical power budget, which is higher for Origins than for JWST, requiring the addition of two extra panels to the current five-panel array. This larger array can be packaged for launch as shown in Fig. 15. This thicker stack does encroach on the keep-put zones defined by the Ariane 5 launch vehicle. This small envelope violation is not a "show stopper" for 


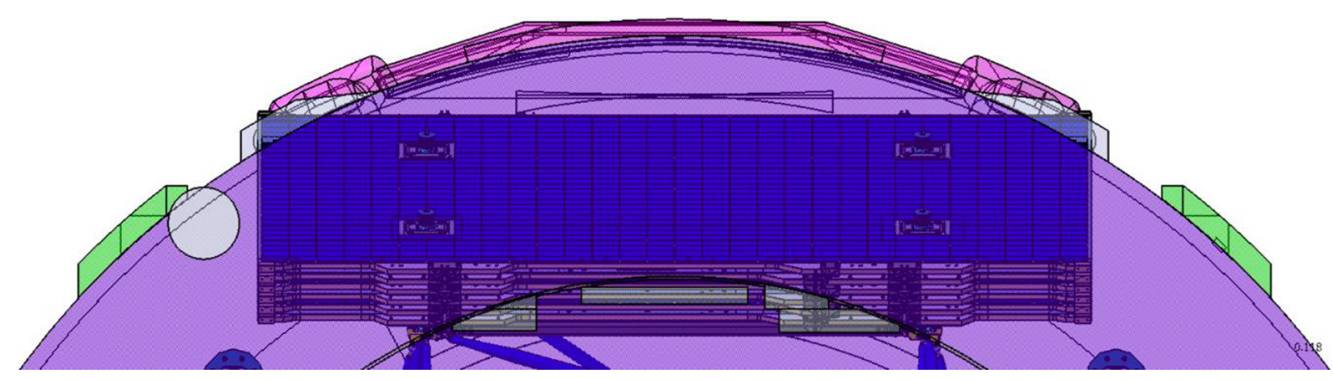

Fig. 15 Stowed seven-panel solar array on the Webb bus that protrudes beyond existing approved Launch Vehicle Solar Array Protrusions. However, it does not protrude beyond existing approved Launch Vehicle Sunshield Protrusions.

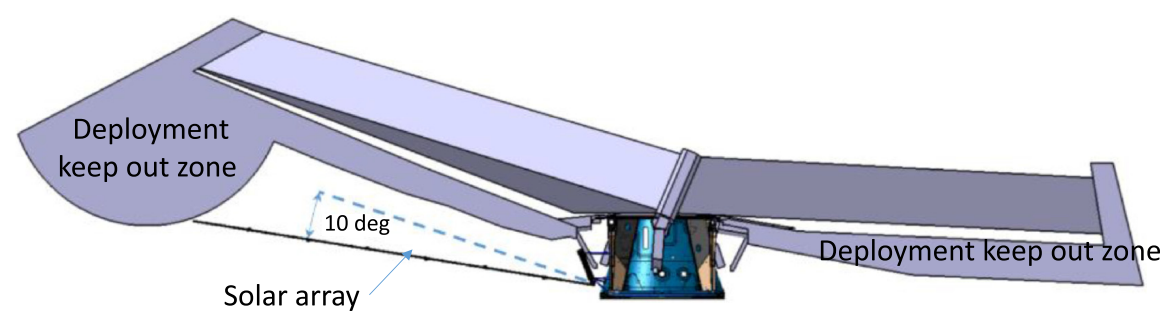

Fig. 16 Deployed envelope for Origins length solar array and adjustment to avoid interference with the aft (momentum) flap.

two reasons. The first is that that envelope violations are small and in family with others that we know of that have been waived for JWST. Second is that when this JWST-derived Origins design might launch, the Ariane 5 will be retired, so a small violation is no reason to reject a concept at this level of development.

We also considered that the longer length of the solar array may have potential interference with the inviolate sunshield deployment envelopes. To accommodate the longer solar array, the angle of deployed solar array had to be adjusted down 10 deg as shown in Fig. 16.

Finally, a mass assessment was made. This mass assessment is based on the JWST mass properties for the reused Webb hardware and study values for the instruments. All told, the Origins wet mass is $\sim 6505 \mathrm{~kg}$, with small mass margins on the JWST legacy hardware and the same mass growth allowance used by the Origins study and reported there for that portion of the hardware. ${ }^{1}$ Our study also indicates that the center of gravity of the alternate architecture is compliant to the requirements of JWST momentum management solution, allowing us to conclude at the conceptual level that once the alternate architecture has reached operating temperature, it can be operated in the same efficient manner as will JWST, allowing reuse of all the apparatus and software of Webb planning and operations.

\section{Summary}

This brief report has shown that the concept of using a modified Webb architecture can meet the thermal needs of Origins. Our results reconfirm the SAFIR studies and Origins study that a large cold telescope is viable. Our alternate architecture for Origins employs three modified MIRI coolers with well understood and demonstrated modifications to achieve the $4.5 \mathrm{~K}$ required for Origins. It is worth noting that baseline Origins design requires a number of state-of-the-art coolers, four, similar to our findings, which call for three. ${ }^{1,2}$ The Origins team approached the design problem in a very different way and came to a similar conclusion about the cryothermal design, namely, that Origins requires only a small number of existing coolers to achieve its mission. In short, $4.5-\mathrm{K}$ class large observatories are viable and possible. The challenge to realizing the thermal performance of Origins is one of architecture, design, and workmanship, not technology. 
By repurposing the Webb architecture for Origins, we have also demonstrated that there are possible observatory architectures that can accommodate telescopes of various spectral ranges and operating temperatures. This exciting possibility opens the way for planned reuse of architecture, design, and hardware. Such a program of programs could provide the needed increase in productivity and launch tempo that could enable a sustainable future for flagship missions such as Webb and Origins.,

\section{Acknowledgments}

This work was supported by Northrop Grumman IR\&D funds. These internal funds supported the system design work and the cryocooler development. The cryocooler work builds upon prior work that was performed for the Jet Propulsion Laboratory, California Institute of Technology, sponsored by the United States Government under a Prime Contract between the California Institute of Technology and NASA. The authors also acknowledge the encouragement of the Origins study office in exploring this idea. We also gratefully acknowledge the anonymous reviewers whose numerous comments and directions helped to make this a much better paper.

\section{References}

1. "Origins Space Telescope documents," https://asd.gsfc.nasa.gov/firs/docs/ (accessed 1 August 2019).

2. M. Dipirro et al., "Origins space telescope cryo-thermal system," J. Astron. Telesc. Instrum. Syst. 7(1), 011009 (2021).

3. J. W. Arenberg et al., "A new paradigm for space astrophysics missions," Proc. SPIE 9143, 914315 (2014).

4. J. W. Arenberg and C. Atkinson, "Ensuring the enduring viability of the space science enterprise," Proc. SPIE 11115, 111150E (2019).

5. J. W. Arenberg et al., "SAFIR architecture concept," Proc. SPIE 5487 (2004).

6. C. F. Lillie and D. R. Dailey, "A mission architecture for future space observatories optimized for SAFIR,” Proc. SPIE 5899, 58990Q (2005).

7. M. Petach, M. Michaelian, and T. Nguyen, "Modifications to the MIRI cryocooler design to provide significant lift in the $2 \mathrm{~K}$ to $4 \mathrm{~K}$ range," IOP Conf. Ser. Mater. Sci. Eng. 755, 012018 (2020).

8. P. A. Lightsey et al., "Stray light overview for the Origins Space telescope," Proc. SPIE 10698, 1069845 (2018).

9. M. DiPirro, "Cryocooling technologies for the Origins Space Telescope," J. Astron. Telesc. Instrum. Syst. 7(1), 011008 (2021).

10. Origins Study Team, "Origins Space Telescope technology development plan," https://asd .gsfc.nasa.gov/firs/docs/Origins Volume2TechDevelopmentPlanREDACTED.pdf (accessed November 2020).

11. J. Raab and E. Tward, "Northrop Grumman aerospace systems cryocooler overview," Cryogenics 50, 572-581 (2010).

12. D. Durand et al., "Mid infra red instrument (MIRI) cooler subsystem design," Cryocoolers 15, 7-12 (2009).

13. M. Petach and M. Michaelian, "Mid infrared instrument (MIRI) cooler cold head assembly acceptance testing and characterization," Cryocoolers 18, 11-17 (2014).

14. M. Petach et al., "Mid infrared instrument (MIRI) cooler compressor assembly characterization," Cryocoolers 19, 1-8 (2016).

15. T. Nguyen et al., "10 K pulse tube cooler," in Presented 14th Int. Cryocooler Conf., Annapolis, MD, pp. 27-31 (2006).

16. J. Arenberg et al., "Thermal considerations and architecture for Origins Space Telescope," Proc. SPIE 10698, 10698 (2018).

17. National Institute of Standards and Technology, "NIST REFROP9," https:/www.nist.gov/ system/files/documents/srd/REFPROP9.PDF (accessed 19 November 2020).

18. M. Petach et al., "Test of a sub-4K mechanical cooler for IXO and other space based sensors," Bull. Am. Astron. Soc. 41, 347 (2009). 
19. S. Backhaus, E. Tward, and M. Petach, "Traveling-wave thermoacoustic electric generator," Appl. Phys. Lett. 85(6), 1085-1087 (2004).

20. T. Bradshaw and A. Orlowska, "Mechanical cooling systems for use in space," Proc. Inst. Mech. Eng. G 207, 21-25 (1993).

21. T. Bradshaw, A. Orlowska, and J. Hieatt, "Development status of a $2.5 \mathrm{~K}-4 \mathrm{~K}$ closed cycle cooler suitable for space use," in Paper presented 24th Int. Conf. Environ. Syst. and 5th Eur. Symp. Space Environ. Control Syst., Friedrichshafen, Germany (1994).

Jonathan W. Arenberg received his BS, MS, and PhD degrees from the University of California, Los Angeles. He is a chief engineer for Space Science Missions at Northrop Grumman Space Systems, working on the Chandra X-ray Observatory, Starshade, JWST, technology development, and numerous mission studies. He is widely published and is an SPIE fellow.

John Pohner received his BME, MSME, and PhD degrees from Georgia Tech. He is the Northrop Grumman Technical fellow in the domain of spacecraft thermal control. He has 35 years of experience and was the NG thermal design lead on JWST from 2002 to 2010. He was a principal investigator for IR\&D programs developing advanced heat transport technologies. He was also instructor of the 12-week UCLA Extension class "Spacecraft Thermal Management."

Michael Petach is a senior staff engineer with more than 29 years of experience in the design, analysis, and testing of pulse tube cryocoolers and thermoacoustic power converters. He has held a principal role in the development of the MIRI cryocooler, a traveling wave thermoacoustic electric power generator, and a two-stage thermoacoustic heat engine for operation at reduced hot end temperatures.

Ryan Hall received his BS degree in mechanical engineering from the University of California, Santa Barbara and his MS degree in mechanical engineering from the University of Southern California. He has more than 14 years of experience in the aerospace industry and is a specialist in spacecraft mechanical design and integration. He has spent the last decade making contributions to the JWST while working at Northrop Grumman Space Systems.

Jeffrey Bautista received his bachelor's degree in mechanical engineering and his master's degree in systems architecting and engineering both from the University of Southern California. He has more than 24 years of experience in the aerospace industry and experience in vehicle systems engineering. He has contributed to systems engineering effort, with a focus on mass properties, while supporting JWST for more than 10 years.

Mark Michaelian received his BS, MS, and PhD degrees in aerospace engineering from the University of Southern California. He has more than 19 years of experience in the aerospace industry and has contributed to the JWST using his background in cryocooler technologies and propulsion.

Tanh Nguyen is a retired Northrop Grumman Technical fellow for cryocoolers and thermophysics devices. 